\title{
Carrier and refractive index dynamics in core-shell nanolasers grown on silicon during spontaneous and stimulated emission
}

Tomasz J. Ochalski, Juan S. D. Morales, Shumithira Gandan, Diana L. Huffaker, Hyunseok Kim, et al.

Tomasz J. Ochalski, Juan S. D. Morales, Shumithira Gandan, Diana L. Huffaker, Hyunseok Kim, Liam O'Faolain, "Carrier and refractive index dynamics in core-shell nanolasers grown on silicon during spontaneous and stimulated emission," Proc. SPIE 11081, Active Photonic Platforms XI, 110812B (12 September 2019); doi: 10.1117/12.2529494

SPIE Event: SPIE Nanoscience + Engineering, 2019, San Diego, California, United States 


\title{
Carrier and refractive index dynamics in core-shell nanolasers grown on silicon during spontaneous and stimulated emission
}

\author{
Tomasz J. Ochalski ${ }^{\mathrm{a}, \mathrm{b}}$, Juan S.D. Morales ${ }^{\mathrm{a}, \mathrm{b}}$, Shumithira Gandan ${ }^{\mathrm{a}, \mathrm{b}}$, Diana L. Huffaker ${ }^{\mathrm{c}}$, Hyunseok \\ Kim $^{\mathrm{c}}$, Liam O'Faolain ${ }^{\mathrm{a}, \mathrm{b}}$ \\ ${ }^{a}$ Cork Institute of Technology, Centre for Advanced Photonics \& Process Analysis, Rosa Av. Cork, \\ T12P928 Ireland; ${ }^{\text {b}}$ Tyndall National Institute, Lee Maltings Complex, Dyke Parade, Cork, 12R5CP \\ Ireland; 'Integrated Nano Materials Core Lab, California Nano Systems Institute, University Of \\ California Los Angeles 90095, CA USA
}

\begin{abstract}
In this work, we experimentally study the carrier and refractive index dynamics of InGaAs nanopillar grown on a Si on insulator (SOI) substrate. The recombination process of the InGaAs NP is characterized with different optical techniques. Temperature dependent photoluminescence (PL) at $0.5 \mathrm{~mW}$ excitation power is carried out to determine the influence of temperature on carrier dynamics. The radiative recombination lifetime has been studied at $7 \mathrm{~K}$ from time-resolved photoluminescence (TRPL) experiments at a certain excitation power. The optimal combination of pitch (separation between NPs) and diameter in the growth process of this nanostructure has also been measured. These results will contribute to further optimization of the InGaAs nanolaser for integration of III-V optoelectronics on SOI substrates.
\end{abstract}

Keywords: Time resolved photoluminescence, nanolasers, SOI, III-V on Silicon

\section{INTRODUCTION}

The applications of (nano)lasers are numerous and extremely varied. It is easy to find coherent light emitters in medicine, food science, manufacturing companies, military sector and research. III-V materials possess high gain value, direct band gap, and easy band gap tunability by varying the alloy component. The major problem of III-V materials growth in silicon is the lattice mismatch in the interface, which produces dislocation and poor performance. In III-V semiconductor nanowires (NWs) and nanopillars (NPs), the lattice mismatch can be fully relaxed over a small number of monolayers because of its small footprint. Moreover, NWs/NPs offer significant potential for nanoscale laser source due to the quasi-one-dimensional structure, which facilitates a natural Fabry-Perot resonator cavity and optical gain medium simultaneously ${ }^{1,2}$. Kim et al. ${ }^{3}$ work observed stimulated light emitted from an InGaAs/InGaP core-shell NWs array at room temperature. The coherent light was resulting from the resonance in a one-dimensional photonic crystal. In this publication, the light emitted was also coupled into silicon on insulator waveguide. On the other hand, Chen et al. ${ }^{2}$ group published the emission of coherent light from a single InGaAs/GaAs core-shell NP optically pumped at $4 \mathrm{~K}$. Strong confined helically propagating modes generated the stimulated output light. Chen et al. also modelled the relation between the NP dimension, wavelength emission and field profile for a set of helically propagating modes with FDTD techniques.

*tomasz.ochalski@cit.ie; phone +353 21234 6836; cappa.ie

Active Photonic Platforms XI, edited by Ganapathi S. Subramania, Stavroula Foteinopoulou, Proc. of SPIE Vol. 11081, 110812B · (C) 2019 SPIE · CCC code: 0277-786X/19/\$21

doi: $10.1117 / 12.2529494$ 


\section{NANOLASER STRUCTURE AND EXPERIMENTAL SETUP}

\subsection{Nanolaser growth and structure}

In this paragraph, a brief summary of the growth process for InGaAs NPs published by Kim et al ${ }^{3,4}$ will be presented. The NPs were fabricated by Hyunseok Kim at Department of Electrical Engineering, University of California Los Angeles inside the group led by Diana L. Huffaker. InGaAs NPs are grown on Silicon on insulator (SOI) (111) wafer lightly p-doped with Boron by metalorganic chemical vapour deposition (MOCVD), figure 1. The NPs features, such as diameter and separation between them (pitch), are patterned in a $20 \mathrm{~nm}$ thick silicon nitride (Si3N4) mask for selective area epitaxy, figure $1 \mathrm{~b}$. Before the growth process start, the native oxide is removed by $6: 1$ buffered oxide etch solution for $60 \mathrm{~s}$ and rinsed with DI water.

a)

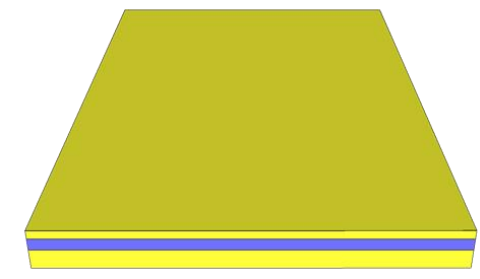

c)

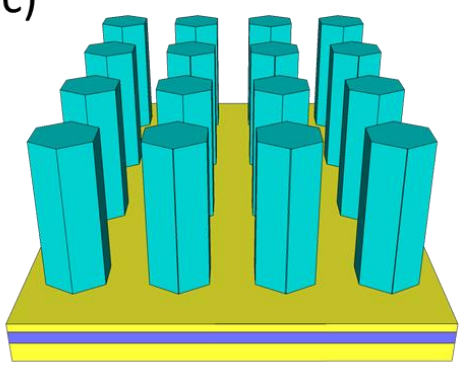

b)

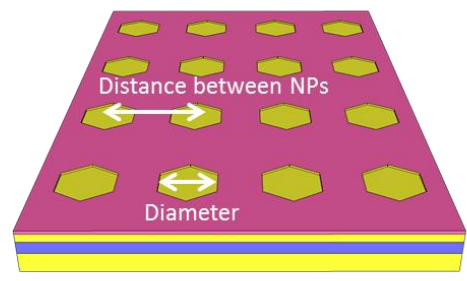

d)

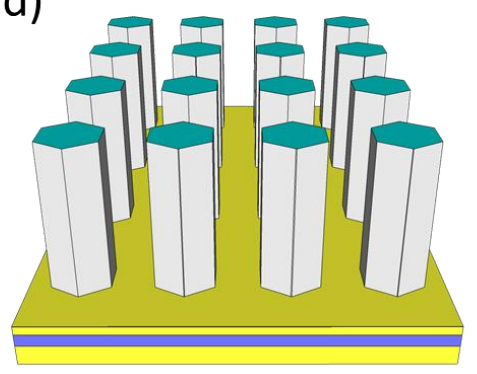

Figure 1. Schematic growth process. a, SOI (111) substrate. b, Si3N4 mask with lithography pattern. c, InGaAs NP growth. d, passivation with InGaP.

Triethylgallium (TEGa), trimethylindium (TMIn), and tertiarybutylarsine (TBAs) are used as precursors and hydrogen as a carrier gas. The sample is first baked for thermal de-oxidation at $860{ }^{\circ} \mathrm{C}$ and 13 minutes in the MOCVD reactor. Then, the temperature is decreased to $690{ }^{\circ} \mathrm{C}$, and the InGaAs nanopillar growth is initiated by flowing TEGa, TMIn, and TBAs simultaneously. The molar flow rates used corresponds to a gas phase composition of $\mathrm{In} /(\mathrm{In}+\mathrm{Ga})=0.257 \mathrm{and} \mathrm{V} / \mathrm{III}$ flow rate ratio of 24.1, figure 1c. The InGaAs/InGaP core-shell NPs are passivated in-situ by growing InGaP shells for $45 \mathrm{~s}$ at $600{ }^{\circ} \mathrm{C}$ with gas phase indium composition $(\mathrm{In} /(\mathrm{In}+\mathrm{Ga}))$ of 0.75 , figure $1 \mathrm{~d}$. Finally, the sample is cooled down with TBAs to prevent desorption.

\subsection{Time resolved PL set-up}

Error! Reference source not found. shows the experimental photo-luminescence set-up to characterise the GaAs NWs and InGaAs NPs. The excitation source is a pulsed Ti-Saphire laser. The wavelength can be tuned from $690 \mathrm{~nm}$ to 1000 $\mathrm{nm}$, and the pulse width is approximately $200 \mathrm{fs}$. The laser beam is guided to a neutral-density filter to have control of the excitation power amount. The micorscope lens is a 50x Olympus silicone achromatic with numerical aperture of 0.65. In order to visualise the sample and the laser beam location, a $660 \mathrm{~nm}$ LED source and CMOS camera is placed in the set-up. The sample emission is guided with beamsplitters to the lens that focuses it on the monochromator. The 
monochromator filters the light and sends it to the femtowatt photodetector, or the streak camera. The sample is placed in a Helium continuous flow liquid Helium cryostat.

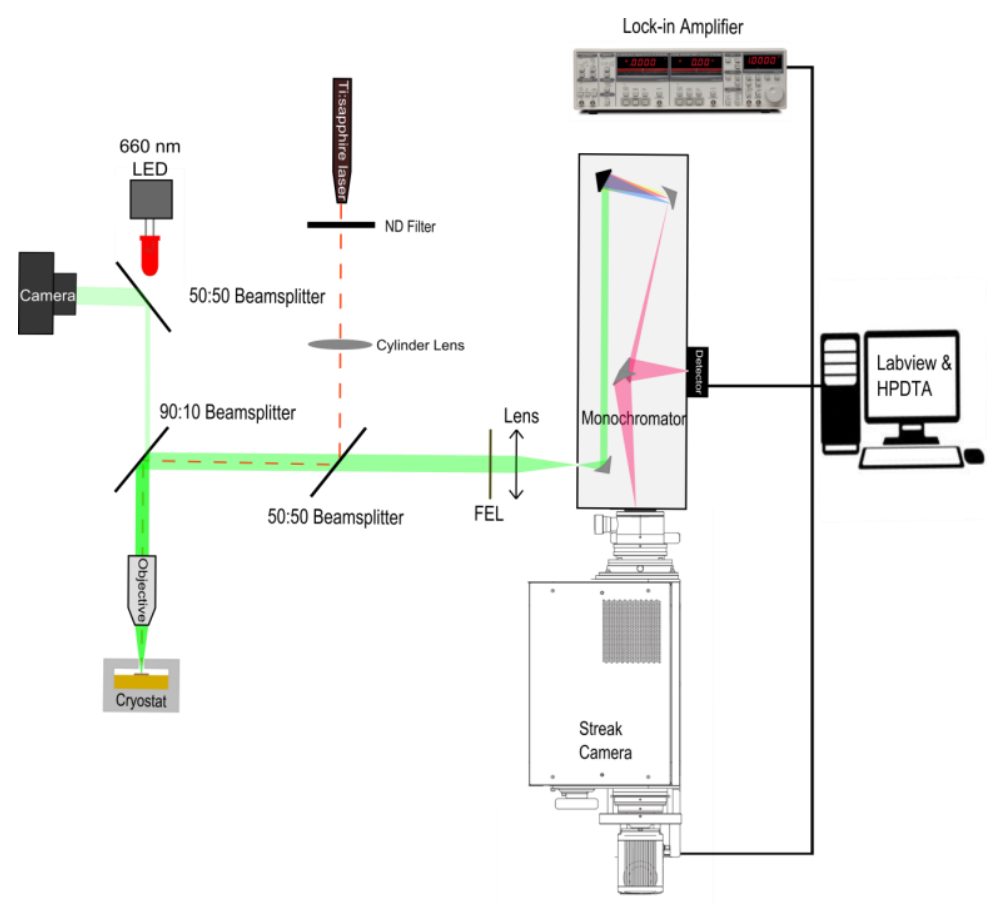

Figure 2. Figure captions are indented 5 spaces and justified. If you are familiar with Word styles, you can insert a field code called Seq figure which automatically numbers your figures.

\section{SPONTANEOUS AND STIMULATED EMISSION FROM INGAAS NANOLASERS}

The emission dynamics of InGaAs NPs was studied for excitation power below and above the lasing threshold. The time evolution for InGaAs NP emission showed a decrease of carrier lifetime with the transition from spontaneous to stimulated carrier recombination process. The optical properties of passivated and unpassivated InGaAs NPs ware also compared. Passivated NPs showed an increase of the carrier lifetime due to the reduction of non-radiative process on the NP surface. Finally, the polarisation study revealed a circular polarisation light for a helical mode propagating inside the InGaAs/InGaP NP.
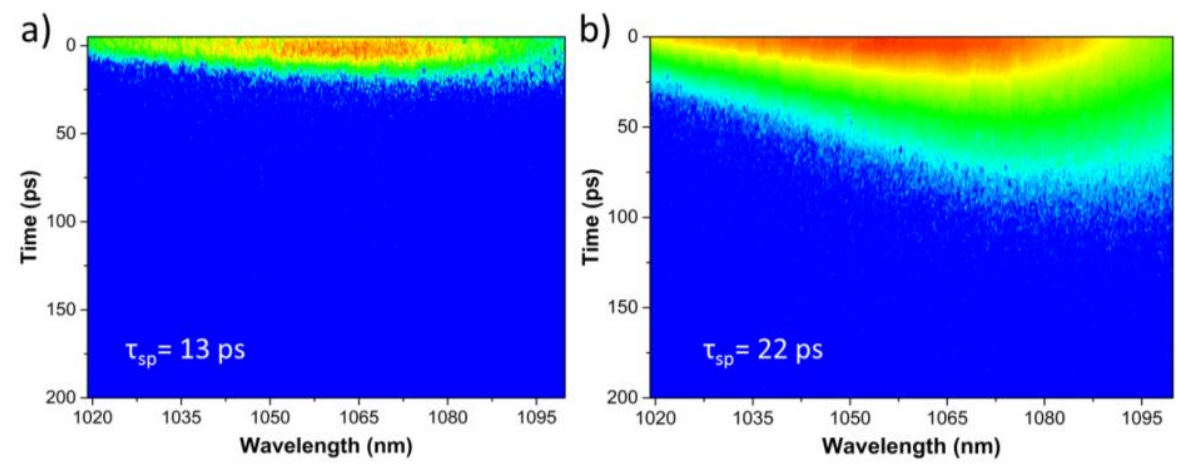

Figure 3. Evolution of time resolved PL under optical pulsed excitation with pump power density corresponding to spontaneous light emission from the unpasivated nonpolar. 
Figures 3 and 4 are presenting time resolved emission measurements below and above lasing threshold respectively. At the fig. $4 \mathrm{~b}$ there is single longitudal lasing mode with clear spectral narrowing and decay time of $6 \mathrm{ps}$ which is at the edge of the system temporal resolution.
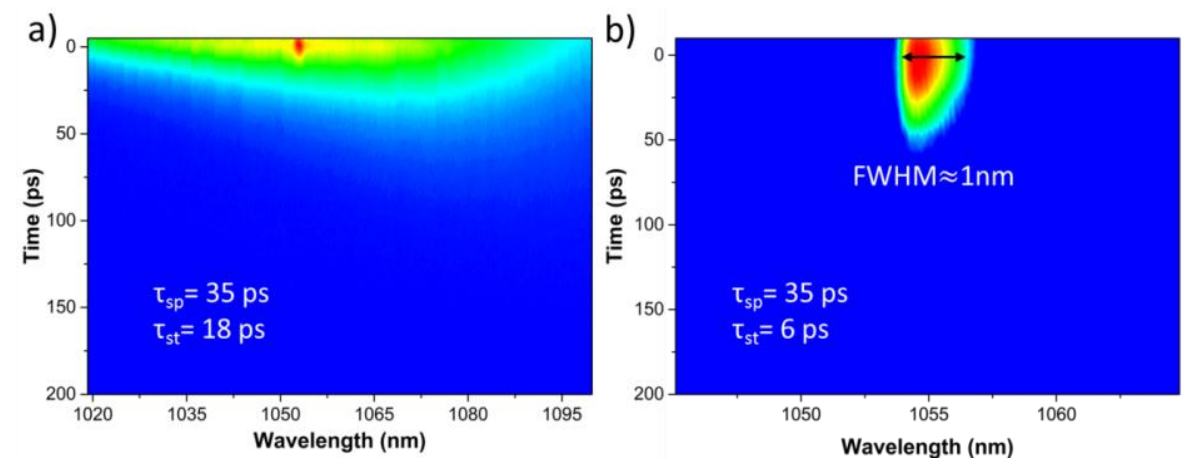

Figure 4. TRPL measurements of InGaAs NPs with $500 \mathrm{~nm}$ pitch and $80 \mathrm{~nm}$ nanohole diameter at $7 \mathrm{~K}$. a, excitation power of 160 $\mathrm{kW} / \mathrm{cm} 2$ (a). Excitation power of $180 \mathrm{~kW} / \mathrm{cm} 2$ with the high-spectral resolution measurement (b).

Passivated InGaAs nanolasers characterize significantly brighter and temporarly longer spontaneous light emission. The examined laser has its F-P mode slightly outside the gain curve. Big longitudal mode spectral separation (in order of 100nm) makes those nanolasers single mode operating devices without any additional processing. Unfortunately the passivated nanolaser F-P mode spectral position is placed at the shoulder of the gain curve.. Due to this fact the laser did not improve threshold value compare to unpassivated laser. Fig. 5 presents Light input light output curve (a) and position of the fast decaying stimulated emission peak (835nm) on the high energy shoulder of the long decaying broad spontaneous emission.
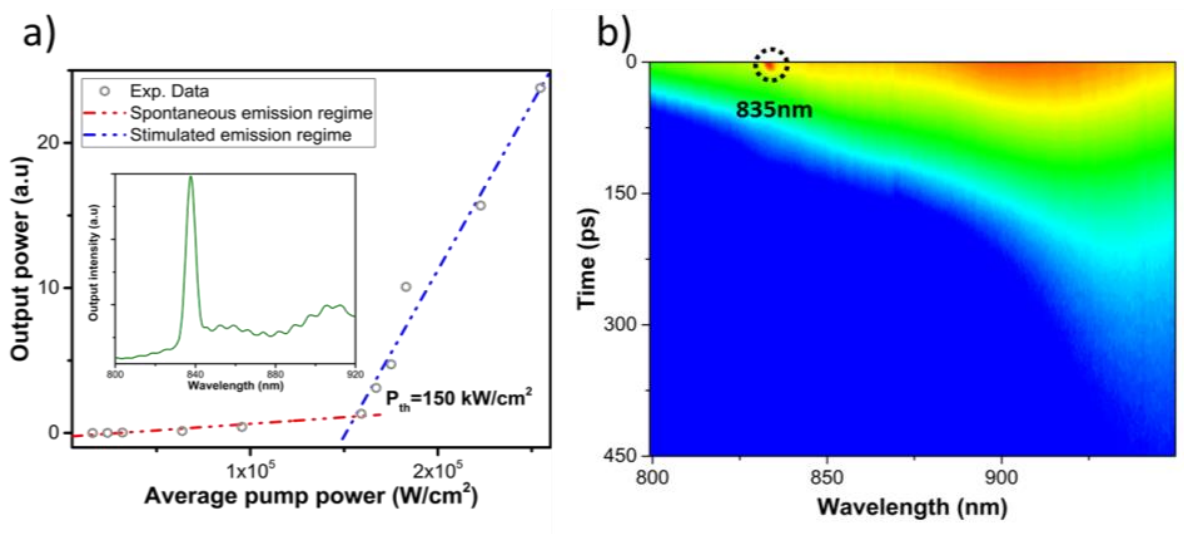

Figure 5. Light input - light output curve for passivated nanolaser structure and optical emission specrtum just above the threshold in the insert (a). Time resolved photoluminescence immage containing two differenf features:

broad long decaying spontaneus emission centered around $925 \mathrm{~nm}$ and spectraly narrow emission, very short decaying F-P single mode just above lasing threshold at $835 \mathrm{~nm}$.

\section{CONCLUSIONS}

The optical properties of passivated and unpassivated InGaAs NPs have been compared in the means of micro-TRPL experiments. The study of carrier lifetime and spectral emission intensity as a function of the excitation power determined the lasing threshold pump power density at the similar level for both unpassivated and passivated NPs. The spontaneous emission intensity for passivated structure is significantly brighter and decays longer, this indicates efficient 
blockage of surface carrier recombination in the NPs. Our experiments also shown that F-P resonance spectral position is extremally critical for reduction lasing threshold thus precise control of the NPs height during growth process is crucial to achieve perfect spectral tuning of the gain curve and F-P resonance.

\section{ACKNOWLEDGMENTS}

We acknowledge financial support from Department of Agriculture, Food and the Marine, Ireland (project reference 15/F679)

\section{REFERENCES}

[1] Chen, R. et al. Nanolasers grown on silicon. Nat. Photonics 5, 170-175 (2011).

[2] Eaton, S. W., Fu, A., Wong, A. B., Ning, C.-Z. \& Yang, P. Semiconductor nanowire lasers. Nat. Rev. Mater. 1, 16028 (2016).

[3] Kim, H. et al. Monolithic InGaAs Nanowire Array Lasers on Silicon-on-Insulator Operating at Room Temperature. Nano Lett. 17, 3465-3470 (2017).

[4] Kim, H., Farrell, A. C., Senanayake, P., Lee, W.-J. \& Huffaker, D. L. Monolithically Integrated InGaAs Nanowires on 3D Structured Silicon-on-Insulator as a New Platform for Full Optical Links. Nano Lett. 16, 1833-1839 (2016). 